“( 2014 IEEE. Personal use of this material is permitted. Permission from IEEE must be obtained for all other uses, in any current or future media, including

reprinting/republishing this material for advertising or promotional purposes, creating new collective works, for resale or redistribution to servers or lists, or reuse of any copyrighted component of this work in other works." 


\title{
Core Loss Computation in a Permanent Magnet Transverse Flux Motor with Rotating Fluxes
}

\author{
Youguang Guo ${ }^{1}$, Senior Member, IEEE, Jianguo Zhu' ${ }^{1}$, Senior Member, IEEE, Haiyan Lu¹, Member, IEEE, Yongjian \\ $\mathrm{Li}^{2}$, Jianxun $\mathrm{Jin}^{3}$, Senior Member, IEEE \\ ${ }^{1}$ Faculty of Engineering and Information Technology, University of Technology Sydney, NSW 2007, Australia \\ ${ }^{2}$ School of Electrical Engineering, Hebei University of Technology, Tianjin 300160, China \\ ${ }^{3}$ School of Automation, University of Electronic Science and Technology of China, Chengdu 611731, China
}

\begin{abstract}
This paper presents the core loss computation in a permanent magnet transverse flux motor (TFM) with soft magnetic composite stator core and mild steel rotor yoke, in which the magnetic fluxes rotate. The computation is based on modified core loss models and finite element magnetic field analysis (FEA). The coefficients for the core loss models are obtained by curve-fitting measurements on samples, and the magnetic flux density patterns in the motor are obtained by time-stepping FEA while operating conditions are considered. The computations of the motor core losses agree with the measured values on the TFM prototype.
\end{abstract}

Index Terms - Core loss, finite element analysis, rotating magnetic flux, soft magnetic composite, transverse flux motor.

\section{INTRODUCTION}

$\mathrm{T}_{\mathrm{t}, \mathrm{sin}}$ RANSVERSE FLUX MOTORS (TFMs) can provide very high torque to volume ratio and hence they have attracted strong research interests in the past three decades [1]-[3]. The structures of these machines are in general complex and have three-dimensional (3-D) magnetic flux paths. The conventional lamination steels are not appropriate or very difficult for construction of TFMs as the magnetic flux must be designed to flow within the lamination plane or excessive eddy current losses would be generated. To overcome this problem, soft magnetic composite (SMC) materials may be employed. SMCs are composed of pure iron particles (about $0.1 \mathrm{~mm}$ in diameter) with very thin insulation coating and hence possess many unique properties such as 3-D magnetic isotropy, very low eddy currents, and prospect of low cost mass production of electrical machines [4]. A few TFM prototypes with SMC cores have been investigated [5]-[6].

For the best use of the material, SMC electromagnetic devices are often designed to operate at $300 \mathrm{~Hz}$ or higher and the core loss is comparable to the copper loss. This is quite different from the conventional laminated steel machines with operating frequency of 50 or $60 \mathrm{~Hz}$, in which the copper loss dominates. Hence, accurate computation of core loss is crucial for developing high performance SMC devices [7]-[10].

This paper presents the computation of core losses of a prototype TFM with SMC stator [5], [11]. Finite element analysis (FEA) is conducted to find out the flux density patterns in each element of the core at different operating conditions. A modified model, which can consider the effect of any type of flux density pattern, is applied to calculate the motor core loss for any given operating condition. The coefficients in the core loss models are obtained by curvefitting the measurements on an SMC sample with a frequency range of $5-1000 \mathrm{~Hz}$ [12]. The calculations agree with the experimental results on the TFM prototype.

Manuscript received March 5, 2014, revised April 14, 2014. Corresponding author: Youguang Guo (e-mail: Youguang.Guo1@uts.edu.au).

\section{MAGNETIC FiELD FEA OF THE TFM PROTOTYPE}

Fig. 1 shows the photos of the studied SMC TFM prototype [5]. The internal stator consists of three stacks of SMC core, which are shifted by 120 electrical degrees to each other, and each is wound by a phase winding of concentrated coil. The external rotor is made of mild steel yoke and permanent magnets (PMs) glued on the inner surface of the yoke. The major dimensions of the motor include $80 \mathrm{~mm}$ of stator outer diameter, $93 \mathrm{~mm}$ of effective stator axial length, and $1 \mathrm{~mm}$ of main air gap length. The motor was designed to operate at 640 W and 1800 rpm with optimum brushless DC control scheme.

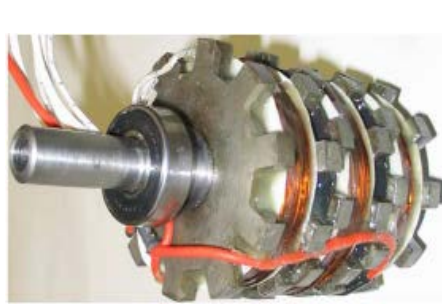

(a)

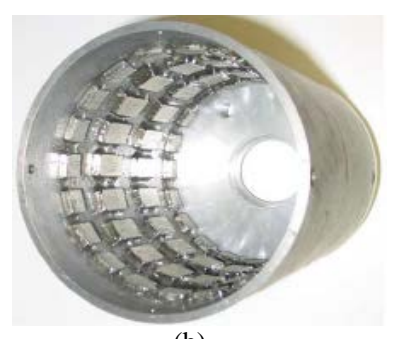

(b)
Fig. 1. Photo of an SMC TFM prototype: (a) stator; (b) rotor

The magnetic flux path in the TFM is predominantly in planes containing the axes but becomes 3-D as it spreads azimuthally to reduce flux densities. For non-linear material properties and accurate flux fringing calculation, a 3-D FEA is required. Taking advantage of the periodical symmetry, only one pole-pair region of the machine, as shown in Fig. 2, needs to be studied. In the figure, A, B and C mark the locations at the middles of SMC stator yoke, SMC stator tooth, and mild steel rotor yoke, respectively. At the two radial boundary planes, the magnetic scalar potential $\left(\varphi_{m}\right)$ obeys

$\varphi_{m}(r, \Delta \theta, z)=\varphi_{m}(r,-\Delta \theta, z)$,

where $r$ and $z$ are the radial and axial coordinates respectively, and $\Delta \theta=18^{\circ}$ mechanical is the angle of one pole pitch. 
3-D magnetic field FEA has been conducted to compute the flux density $(B)$ distribution in the motor under various operating conditions, and work out the $B$ pattern in each element when the rotor rotates. Fig. 3 shows the no-load $B$ loci in a typical stator yoke element (near Point A), in a typical stator tooth element (near Point B), and in a typical rotor yoke element (near Point $C$ ), respectively. As can be seen from Fig. 3(b), the $B$ loci in the SMC stator are basically rotational.

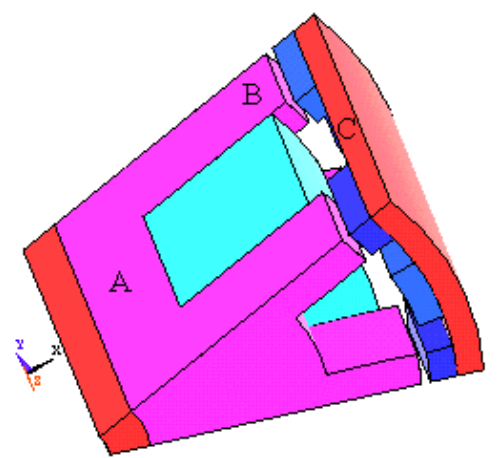

Fig. 2. Region for 3-D magnetic field FEA

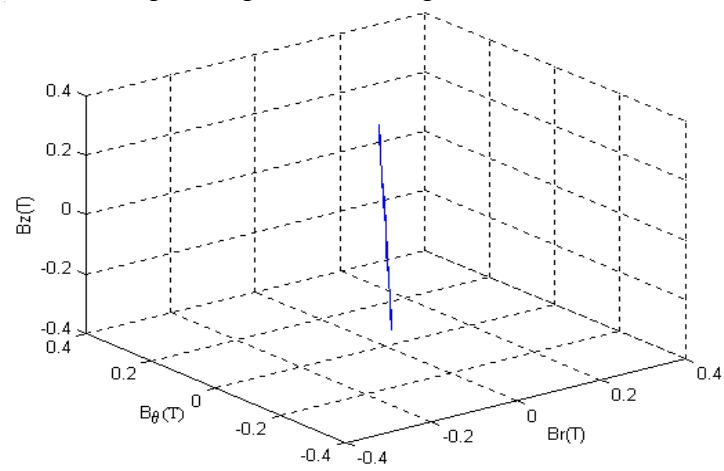

(a)

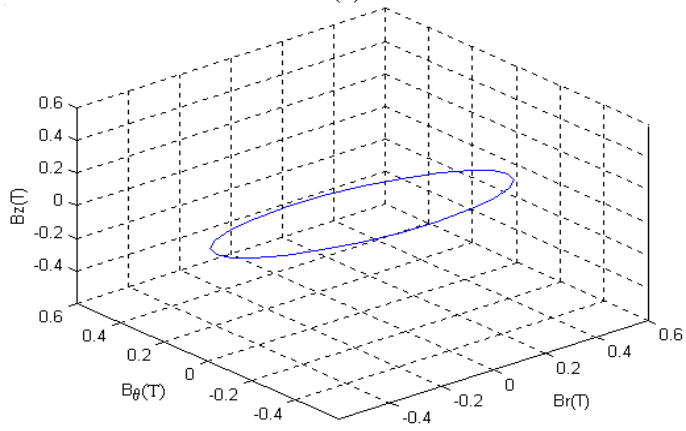

(b)

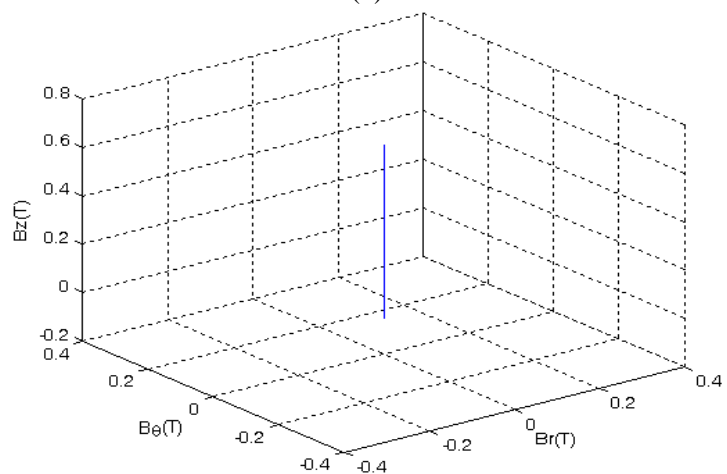

(c)

Fig. 3. No-load $B$ locus in a typical element in (a) stator yoke - point A of Fig. 2, (b) stator tooth - point B of Fig. 2, (c) rotor yoke - Point C of Fig. 2
Fig. 4 shows the variations of the components of flux density versus time (or rotor angle) in the typical elements. The flux density is generally 3-D with non-negligible component in any direction. However, the $B$ in the rotor yoke elements, as shown in Fig. 4(c), are basically one-dimensional (1-D) along the axial direction (z-axis) only, i.e. the magnetic field along the radial ( $r$-axis) or circumferential ( $\theta$-axis) direction is negligible. The calculations also show that the flux density in PMs is almost constant, so the core loss in the PMs is ignored in this work.

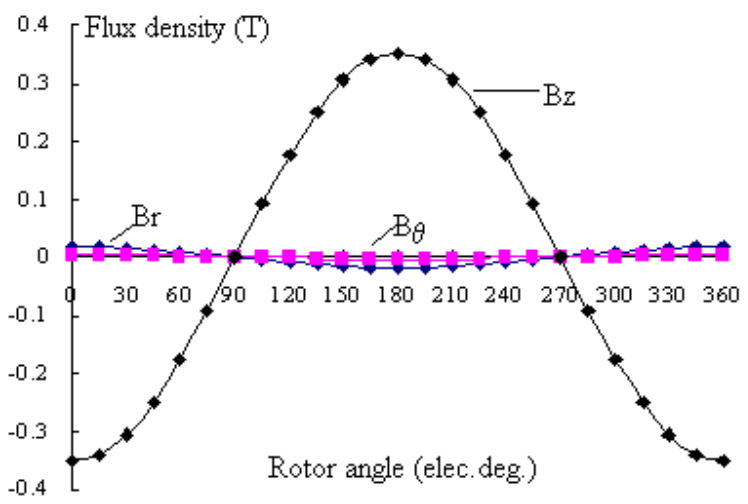

(a)

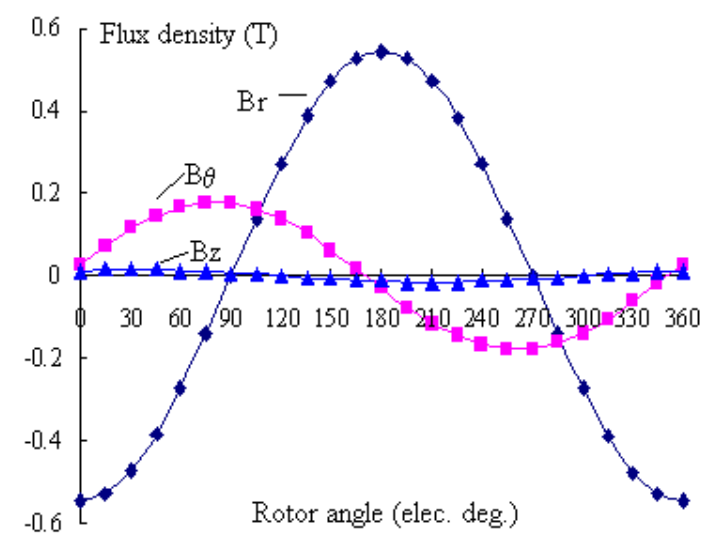

(b)

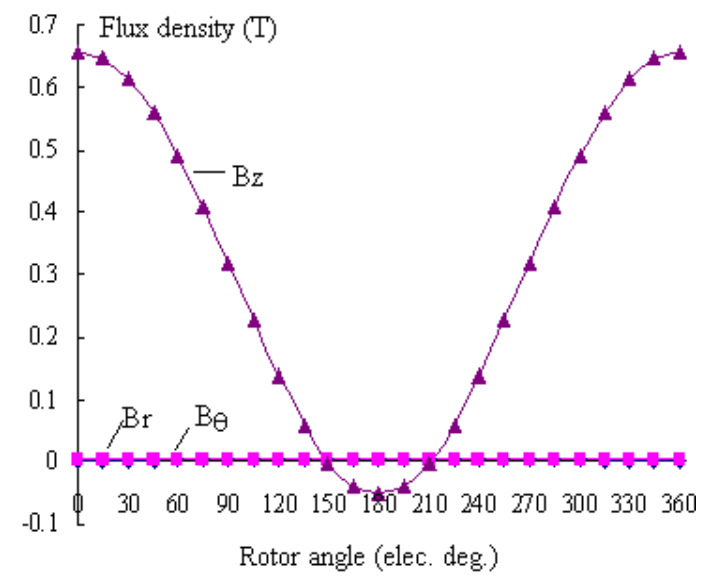

(c)

Fig. 4. No-load $B$ components in a typical element in (a) stator yoke - point A of Fig. 2, (b) stator tooth - point B of Fig. 2, (c) rotor yoke - Point C of Fig. 2 


\section{CORE LOSS MODELS}

To calculate the core losses under rotational $B$ loci, Zhu and Ramsden presented a comprehensive model for calculating the rotational hysteresis loss in laminated sheet steel machines [13]. The formulations are extended to calculate the core losses of this SMC machine, as summarized below.

When the magnetic material is under 1-D sinusoidal $B$ excitation, the alternating core loss $P_{a}$ is computed by

$$
P_{a}=C_{h a} f B_{P}{ }^{h}+C_{e a}\left(f B_{P}\right)^{2}+C_{a a}\left(f B_{P}\right)^{3 / 2},
$$

where $f$ is the excitation frequency, $B_{P}$ is the magnitude of the sinusoidal $B$, and $C_{h a}, C_{e a}, C_{a a}$ and $h$ are alternating core loss coefficients.

When the material is under two-dimensional (2-D) circularly rotating $B$ excitation, the rotational core loss $P_{r}$ is computed by

$$
\begin{aligned}
& P_{r}=P_{h r}+C_{e r}\left(f B_{P}\right)^{2}+C_{a r}\left(f B_{P}\right)^{3 / 2}, \\
& \frac{P_{h r}}{f}=a_{1}\left[\frac{1 / s}{\left(a_{2}+1 / s\right)^{2}+a_{3}^{2}}-\frac{1 /(2-s)}{\left[a_{2}+1 /(2-s)\right]^{2}+a_{3}^{2}}\right], \\
& s=1-\frac{B_{P}}{B_{s}} \sqrt{1-\frac{1}{a_{2}^{2}+a_{3}^{2}}},
\end{aligned}
$$

where $B_{P}$ is the magnitude of the circular $B, B_{s}$ is the material's saturation flux density, and $C_{e r}, C_{a r}, a_{1}, a_{2}$ and $a_{3}$ are rotational core loss coefficients.

When the material is under 2-D elliptically rotating $B$ excitation, the core loss $P$ is computed by

$$
P=P_{r} R_{B}+\left(1-R_{B}\right)^{2} P_{a},
$$

where $R_{B}=B_{\text {min }} / B_{\text {maj }}$ is the axis ratio, $B_{\text {min }}$ and $B_{\text {maj }}$ are the values of the major and minor axes of the ellipse respectively, and $P_{r}$ and $P_{a}$ are the corresponding rotational and alternating core losses when $B_{P}=B_{m a j}$.

\section{CORE LOSS CALCULATION IN THE TFM PROTOTYPE}

\section{A. No-load Core Loss of SMC Stator Core}

As described in Section II, the $B$ pattern in each element can be obtained by time-stepping FEA. In general, the $B$ pattern is an irregular 3-D loop. The 1-D alternating and 2-D rotating loci can be seen as special cases of the 3-D loop.

In each element, the flux density can be divided into three components along the $r$-, $\theta$ - and $z$ - axes, and each component can be expressed by a series of harmonics using Fourier series. It can be verified mathematically that each harmonic forms an elliptical loop in a 2-D plane. Therefore, equations (2)-(6) can be used to calculate the core loss caused by each harmonic in each element. The coefficients in the formulae can be derived by curve-fitting the measurements on a cubic SMC sample under 1-D sinusoidal $B$ excitations and 2-D circular excitations [12].
It can be derived mathematically that the total loss is the sum of all the harmonic losses. Then the core loss in the SMC stator $P_{s m c}$ is

$P_{s m c}=\sum_{e=1}^{N e} \sum_{k=1}^{\infty}\left[P_{r k} R_{B k}+\left(1-R_{B k}\right)^{2} P_{a k}\right]$,

where $\mathrm{Ne}$ is the number of SMC elements used for conducting the FEA, $P_{r k}$ and $P_{a k}$ are respectively the rotational and alternating core losses under the $k$-th harmonic of $B$, and $R_{B K}$ is the axis ratio of the $k$-th $B$ harmonic.

Take the element at point B (stator tooth) as an example. Using the discrete Fourier transform (DFT), the harmonics of the flux density components, $B_{r}, B_{\theta}$ and $B_{z}$, can be derived as

$$
\begin{aligned}
& B_{r} \approx-0.5458 \cos \theta+0.0012 \sin \theta+0.0015 \cos 3 \theta, \\
& B_{\theta} \approx 0.0297 \cos \theta+0.1759 \sin \theta \\
& B_{z} \approx 0.0131 \cos \theta+0.0066 \sin \theta
\end{aligned}
$$

For the fundamental component:

$$
\begin{aligned}
& B_{m a j 1}=\sqrt{0.5458^{2}+0.0297^{2}+0.0131^{2}}=0.5466(\mathrm{~T}), \\
& B_{\min 1}=\sqrt{0.0012^{2}+0.1759^{2}+0.0066^{2}}=0.1760(\mathrm{~T}), \\
& R_{B_{1}}=B_{\min 1} / B_{m a j 1}=0.322 .
\end{aligned}
$$

Therefore, the locus of the fundamental component of the flux density is of an elliptically rotating pattern within a plane, which is not parallel to any coordinate axis. For the $3^{\text {rd }}$ harmonic: $B_{\text {maj3 }}=0.0015, B_{\min 3}=0$, and $R_{B 3}=0$ and thus it is an alternating flux.

In summary, the flux density locus in each element is calculated first by the FEA of magnetic fields. Then, the ratio of the major and minor axes of the fundamental and harmonic components is calculated. Using equations (2)-(7), the core loss in the SMC stator is computed as $29 \mathrm{~W}$.

\section{B. No-load Core Loss of Mild Steel Rotor Yoke}

In the mild steel rotor yoke, the $B$ patterns are alternating along the $z$ direction only, so only (2) may be required. The alternating coefficients are obtained by curve-fitting the data provided by the material manufacturer, which were measured by using a ring sample with 1-D sinusoidal excitation.

An approximate method by analytical formula in combination with the static magnetic field analysis (ignoring the effect of eddy currents) was proposed to predict the core loss in the mild steel rotor yoke. The following assumptions were made: (i) only the eddy current loss is considered since it is much larger than the hysteresis and anomalous components; (ii) the magnetic fields alternate in the axial direction and the induced eddy currents flow circumferentially, which is true in the middle of the yoke but not accurate near the ends; and (iii) the magnetic flux density is obtained by solving the static field analysis, which ignores the effect of eddy currents.

The procedure of core loss calculation is: (i) solving the 
magnetic field distributions of the motor at various rotor positions, (ii) obtaining the flux density curve against rotor angle or time in each element, $i$, (iii) expanding the curve into a series of sine waves by DFT and obtain the peak value of flux density, $B_{P k i}(\mathrm{k}=1,2,3, \ldots)$, (iv) calculating the eddy current loss $P_{e i}$ in $\mathrm{W} / \mathrm{kg}$ for each element by

$$
P_{e i}=C_{e} \sum_{k}\left(k f B_{P k i}\right)^{2}, C_{e}=k_{e} \frac{\pi^{2} t_{r k}^{2} \sigma}{6 \rho_{m}}
$$

where $f$ is the frequency, $B_{P k i}$ the peak flux density of the $k$-th harmonic in element $i, t_{r k}$ the rotor yoke thickness, $\sigma$ the electrical conductivity, $\rho_{m}$ the mass density, and $k_{e}$ a correction factor according to the experiment results, and (v) calculating the total loss $P_{e}$ by summing the loss in each element as

$$
P_{e}=\sum_{i} P_{e i} \rho V_{i}
$$

where $V_{i}$ is the volume of element $i$.

The core loss in the rotor yoke was calculated by using (14) and (15) as $34.3 \mathrm{~W}$, larger than the SMC stator core loss of 29 $\mathrm{W}$. The calculated total core loss is $63.3 \mathrm{~W}$, agreeing with the measured result in Section V.

\section{Core Loss under Load}

The magnetic field distributions were solved with both PM and stator current excitations. At the rated load and the optimum brushless DC control, the stator core loss was computed as $56.5 \mathrm{~W}$ and the rotor yoke core loss was $63.8 \mathrm{~W}$, and hence the total core loss was $120.3 \mathrm{~W}$.

\section{CORE LOSS MEASUREMENT IN THE TFM PROTOTYPE}

To measure the core loss of the TFM prototype, a DC motor is used as the prime mover. The power fed into the DC motor is measured when it is stand-alone and coupled to the TFM, respectively. The major parameters of the DC driving motor, such as armature resistance, brush voltage drop and emf constant are determined from a series of measurements by the curve fitting technique and the fed electromagnetic power is calculated. The difference of the electromagnetic power at the two conditions gives the total of the core loss and mechanical loss of the tested machine [5].

The mechanical loss of the TFM is measured by replacing the SMC stator with a wood tube (to imitate the windage) and then repeating the previous test procedure. It is assumed that the TFM rotor causes the same mechanical loss with the wooden tube or with the SMC stator if running at the same speed. Then the core loss is obtained by subtracting the mechanical loss from the total of the core loss and mechanical loss [5].

The core loss at no-load was measured as $68.2 \mathrm{~W}$ by using the dummy rotor method, which is $4.9 \mathrm{~W}$ larger than the calculated value. The $7.2 \%$ error may be caused by the losses in the endplates and PMs, which were not calculated. The assumption that the flux density in the rotor yoke is at the Zaxis may also cause some error.

Based on various power and loss measurements and the assumption that the mechanical loss at any load is the same for a particular speed, the motor core loss at the rated load was derived as $131.2 \mathrm{~W}$, which is $8.3 \%$ larger than the calculation.

\section{CONCLUSION}

This paper presents the core loss calculation in a transverse flux motor with soft magnetic composite stator and mild steel rotor yoke, based on magnetic field finite element analysis. The presented approaches can deal with various magnetic excitations such as 1-D alternating, 2-D circularly or elliptically rotating, and even 3-D irregular patterns of magnetic flux density. The approaches can also be easily adapted for other types of electrical machines with other magnetic materials.

\section{REFERENCES}

[1] H. Weh and H. May, "Achievable force densities for permanent magnet excited machines in new configuration,” Proc. Int. Conf. on Electrical Machines, Munich, Germany, 1986, pp.1107-1111.

[2] E. Schmidt, "Finite element analysis of a novel design of a three phase transverse flux machine with an external rotor," IEEE Trans. Magn., vol. 47, no. 5, pp. 982-985, 2011.

[3] J. Shin, T. Koseki, and H. Kim, "Proposal of double-sided transverse flux linear synchronous motor and a simplified design for maximum thrust in nonsaturation region," IEEE Trans. Magn., vol. 49, no. 7, pp. 4104-4108, 2013.

[4] Höganäs AB, Soft Magnetic Composite - Somaloy $®$ materials, available at www.hoganas.com/Powder-Applications/SoftMagneticComposites. Accessed on 4 March 2014

[5] Y. G. Guo, J. G. Zhu, P. A. Watterson, and W. Wu, "Development of a permanent magnet transverse flux motor with soft magnetic composite core,” IEEE Trans. Energy Conver., vol. 21, no. 2, pp. 426-434, 2006.

[6] J. Doering, G. Steinborn, and W. Hofmann, "Torque, power, losses and heat calculation of a transverse flux reluctance machine with soft magnetic composite materials and disc-shaped rotor," Proc. IEEE $5^{\text {th }}$ Annual Energy Conversion Congress \& Expo, Denver, USA, 2013, pp. 4326-4333.

[7] Y. G. Guo, J. G. Zhu, H. Y. Lu, Z. W. Lin, and Y. J. Li, "Core loss calculation for soft magnetic composite electrical machines," IEEE Trans. on Magn., vol. 48, no. 11, pp. 3112-3115, 2012.

[8] C. Appino, O. Bottauscio, O. de la Barrière, F. Fiorillo, A. Manzin, and C. Ragusa, "Computation of eddy current losses in soft magnetic composite," IEEE Trans. on Magn., vol. 48, no. 11, pp. 3470-3473, 2012.

[9] A. Bordianu, O. de la Barrière, O. Bottauscio, M. Chiampi, and A. Manzin, "A multiscale approach to predict classical losses in soft magnetic composites," IEEE Trans. on Magn., vol. 48, no. 4, pp. 15371540, 2012.

[10] A. Chebak, P. Viarouge, and J. Cros, "Analytical computation of the full load magnetic losses in the soft magnetic composite stator of high-speed slotless permanent magnet machines," IEEE Trans. on Magn., vol. 45, no. 3, pp. 952-955, 2009.

[11] J. G. Zhu, Y. G. Guo, Z. W. Lin, Y. J. Li, and Y. K. Huang, "Development of PM transverse flux motors with soft magnetic composite cores," IEEE Trans. on Magn., vol. 47, no. 10, pp. 43764383, 2011.

[12] Y. J. Li, J. G. Zhu, Q. X. Yang, Z. W. Lin, Y. G. Guo, and C. Zhang, "Study on rotational hysteresis and core loss under three-dimensional magnetisation,” IEEE Trans. on Magn., vol. 47, no. 10, pp. 3520-3523, 2011.

[13] J. G. Zhu and V. S. Ramsden, "Improved formulations for rotational core losses in rotating electrical machines," IEEE Trans. on Magn., vol.34, no.4, pp. 2234-2242, 1998. 